\title{
Centro de operações de emergência na COVID-19: a experiência do município do Rio de Janeiro
}

\author{
Débora Medeiros de Oliveira e Cruz, ${ }^{1}$ Luciana Freire de Carvalho, ${ }^{1}$ Carolina Monteiro da Costa, ${ }^{1}$ \\ Gislani Mateus Oliveira Aguilar, ${ }^{1}$ Valéria Saraceni, ${ }^{1}$ Oswaldo Gonçalves Cruz, ${ }^{2}$ Betina Durovni, ${ }^{1}$ \\ Daniel Ricardo Soranz ${ }^{1}$ e Márcio Henrique de Oliveira Garcia ${ }^{1}$
}

Como citar Oliveira e Cruz DM, Carvalho LF, Costa CM, Aguilar GMO, Saraceni V, Cruz OG, et al. Centro de operações de
emergência na COVID-19: a experiência do município do Rio de Janeiro. Rev Panam Salud Publica. 2022;46:e9.
https://doi.org/10.26633/RPSP.2022.9

RESUMO

O presente artigo descreve a experiência de implantação de um centro de operações de emergência (COE) para coordenação da resposta à pandemia de COVID-19 no município do Rio de Janeiro, Brasil. Seguindo o modelo de gestão de emergências em saúde pública preconizado pela Organização Mundial da Saúde (OMS), este centro de caráter temporário (COE COVID-19 RIO) foi ativado em janeiro de 2021. O relato foi estruturado com base em cinco eixos temáticos: arcabouço legal; estrutura, planos e procedimentos; articulações institucionais; informações em saúde para apoio decisório; e comunicação de risco. Entre os avanços importantes relacionados ao estabelecimento desta iniciativa, destacaram-se ganhos em governança para a organização do enfrentamento à COVID-19, aumento da sinergia entre setores e instituições, maior compartiIhamento de informações em relação às medidas de prevenção e controle da doença, inovação nas análises epidemiológicas e ganhos na transparência e oportunidade na tomada de decisões. Concluiu-se que, mesmo sendo concebido em estágio avançado da pandemia na cidade, o COE COVID-19 RIO teve papel relevante na estruturação da resposta. Ainda, apesar do caráter temporário do COE, a experiência mostrou-se como importante legado para a condução de futuras emergências em saúde pública no município do Rio de Janeiro.

Palavras-chave Centro de operações de emergência; COVID-19; capacidade de resposta ante emergências.

Com o primeiro caso confirmado em 26 de fevereiro de 2020 (1), a COVID-19 no Brasil se configurou como um grande desafio para o sistema de saúde devido à alta incidência de casos, ampla distribuição geográfica e elevada mortalidade, implicando na necessidade de melhoria do acesso e qualidade do cuidado ofertado, da vigilância epidemiológica e das estratégias de controle $(2,3)$. Mais recentemente, somaram-se a esse contexto a circulação de variantes virais de preocupação $(4,5)$ e dificuldades no processo de aquisição de imunobiológicos e testagem de casos suspeitos.

Secretaria Municipal de Saúde do Município do Rio de Janeiro, Rio de Janeiro (RJ), Brasil. $₫$ Débora Medeiros de Oliveira e Cruz, debora.sanitarista@gmail.com
Na vigência de emergências em saúde pública — situações estabelecidas no Regulamento Sanitário Internacional (RSI) que demandam o emprego oportuno de medidas de prevenção e controle - , a Organização Mundial da Saúde (OMS) recomenda a ativação de centros de operações que modelem a gestão de emergência, reconhecendo a necessidade de respostas coordenadas para o enfrentamento de ameaças e consequências para a saúde pública (6-9). Seguindo as recomendações da OMS, em 22 de janeiro de 2020, o Ministério da Saúde ativou um centro de operações de emergência (COE) para resposta à pandemia pelo

2 Fundação Oswaldo Cruz (Fiocruz), Programa de Computação Científica (PROCC), Rio de Janeiro (RJ), Brasil.

Este é um artigo de acesso aberto distribuído sob os termos da Licença Creative Commons Attribution-NonCommercial-NoDerivs 3.0 IGO, que permite o uso, distribuição e reprodução em qualquer meio, desde que o trabalho original seja devidamente citado. Não são permitidas modificações ou uso comercial dos artigos. Em qualquer reprodução do artigo, não deve haver nenhuma sugestão de que a OPAS ou o artigo avaliem qualquer organização ou produtos específicos. Não é permitido o uso do logotipo da OPAS. Este aviso deve ser preservado juntamente com o URL original do artigo. Crédito do logotipo e texto em acesso aberto: PLoS, sob licença Creative Commons Attribution-Share Alike 3.0 Unported 
novo coronavírus (9). Coordenado pela Secretaria de Vigilância em Saúde, o COE COVID-19 foi implantado em momento anterior à detecção dos primeiros casos no país. Apesar de a iniciativa ter se dado em momento bastante oportuno na esfera federal, o protagonismo desse centro na condução nacional do enfrentamento fragilizou-se a partir de abril de 2020, com a saída de importantes lideranças gestoras do Ministério da Saúde. Nesse sentido, intensificou-se a necessidade de suprir as demandas regionais de planejamento, coordenação e resposta à COVID-19.

Por sua vez, no município do Rio de Janeiro, os primeiros doentes foram identificados no mês de março de 2020, sendo registrados, até o final desse mesmo ano, aproximadamente 193 mil casos de COVID-19 e 18 mil óbitos. A decisão de estruturar um centro para gestão dessa emergência no município foi concebida em dezembro de 2020, durante a transição do governo municipal. Nesse período, iniciou-se uma agenda intensa de cooperação entre a Secretaria Municipal de Saúde do Rio de Janeiro (SMS-Rio) e técnicos do escritório brasileiro da Organização Pan-Americana da Saúde (OPAS) para a concepção de um centro para comando e resposta unificada à pandemia na cidade.

$\mathrm{O}$ presente relato tem como objetivo apresentar a experiência da implantação do COE COVID-19 RIO, que teve como referência o documento da OMS Framework for a Public Health Emergency Operations Centre (10). São abordados os aspectos legais relacionados ao funcionamento do COE COVID-19 RIO, sua estrutura, planos e procedimentos desenvolvidos, parcerias institucionais estabelecidas, informação em saúde para apoio decisório e condutas relacionadas à comunicação de risco.

\section{ARCABOUÇO LEGAL}

A ativação do COE COVID-19 RIO ocorreu em 4 de janeiro de 2021, a partir da publicação de dois decretos centrais. O primeiro decreto definiu o COE COVID-19 RIO como uma unidade de planejamento e gestão coordenada das ações de enfrentamento à pandemia e instituiu as medidas de proteção à vida — condutas para a prevenção da transmissão de COVID-19, alinhadas com a classificação de risco da cidade do Rio de Janeiro. O segundo decreto instituiu o Comitê Especial de Enfrentamento à COVID-19 (CEEC), composto por representantes de instituições de ensino e pesquisa de relevância nacional. As reuniões mensais do CEEC tiveram importância na avaliação e monitoramento das atividades de controle da doença na cidade, bem como na proposição de recomendações ao COE COVID-19 RIO para o aperfeiçoamento das medidas de proteção à vida.

No âmbito municipal, esses instrumentos delinearam as responsabilidades e os mecanismos de gestão na resposta à pandemia, assegurando o funcionamento do centro e a autonomia necessária para a condução das atividades relacionadas à mitigação da doença.

\section{ESTRUTURA, PLANOS E PROCEDIMENTOS}

Em relação aos aspectos estruturais, o COE COVID-19 RIO foi organizado em uma unidade de comando e pontos focais (figura 1). A coordenação das atividades neste centro esteve a cargo do comando, composto por um coordenador geral, pelo grupo de operações (responsável pela organização e operação técnica das atividades de rotina) e pelo grupo de análises (responsável pela elaboração de informações sobre a situação da doença na cidade). Pontos focais pertencentes a setores chaves da SMS-Rio integraram-se ao COE COVID-19 RIO desenvolvendo atividades específicas no campo de sua atuação.

Como procedimentos de rotina, destacaram-se as reuniões diárias de briefing e debriefing entre o comando e os pontos focais. Esses encontros facilitaram o compartilhamento de informações e experiências tanto para a proposição quanto para o acompanhamento de estratégias de prevenção, controle e assistência à população. Relatórios sobre investigações de surtos, programação das atividades de vacinação, planejamento de abertura e fechamento de leitos para internação e validação de protocolos de testagem de casos nas unidades foram algumas das atividades monitoradas nessas reuniões. Para o gerenciamento das atividades no COE foi utilizada uma matriz de responsabilidades, que apresentava o detalhamento das iniciativas e indicava os responsáveis pela execução e pelos prazos de entrega.

Ações direcionadas para cada nível de alerta foram documentadas no Plano Municipal de Contingência para Enfrentamento da COVID-19, coletivamente elaborado no COE. Cada área técnica organizou seu conjunto de ações de resposta, compatíveis com os níveis de alerta moderado, alto e muito alto, que foram definidos com base em um índice composto pelas taxas de mortalidade e internação por COVID-19 nos diferentes territórios da cidade. É importante destacar que o COE COVID-19 RIO foi estabelecido em momento epidemiológico de alta incidência da doença na cidade e as ações iniciais de resposta foram direcionadas aos níveis de alerta alto e muito alto.

Protocolos e outros documentos técnicos, como o Plano Municipal de Imunização, o Guia de Medidas de Proteção à Vida, o Plano de Comunicação e o Guia de Referência Rápida da Abordagem da COVID-19 na Atenção Primária, foram revisados conjuntamente no COE COVID-19 RIO. A versão atualizada desses documentos pode ser obtida no site https:/ / coronavirus.rio/.

\section{ARTICULAÇÕES INSTITUCIONAIS}

A implantação do COE COVID-19 RIO favoreceu a articulação com diversos representantes de setores da saúde, outras secretarias do governo, universidades, organizações não governamentais e gestores de diferentes esferas. Desdobramentos dessas parcerias fortaleceram o planejamento e a implementação das medidas de prevenção e controle da pandemia na cidade.

Atividades organizadas entre a assessoria de comunicação da SMS-Rio e a coordenação do programa de imunizações facilitaram o acesso às informações pertinentes ao calendário de vacinação, apoiando a divulgação da campanha. A ativação do COE municipal proporcionou maior integração entre as equipes de urgência e emergência e do complexo regulador, trazendo celeridade na abertura e operacionalização de leitos destinados a pacientes graves. A atuação conjunta entre a vigilância sanitária e a vigilância epidemiológica qualificou a investigação de surtos, principalmente nas instituições de longa permanência e escolas, favorecendo a redução da transmissão e, em última instância, menor ocorrência de casos graves e óbitos.

Intersetorialmente, foram desenvolvidos junto à Secretaria Municipal de Educação protocolos para retorno seguro às aulas e identificação e notificação de surtos, visando oportunizar a atuação das equipes de vigilância no ambiente escolar. 
FIGURA 1. Estrutura organizacional do Centro de Operações de Emergência COVID-19 RIO: comando e pontos focais da Secretaria Municipal de Saúde ${ }^{a}$

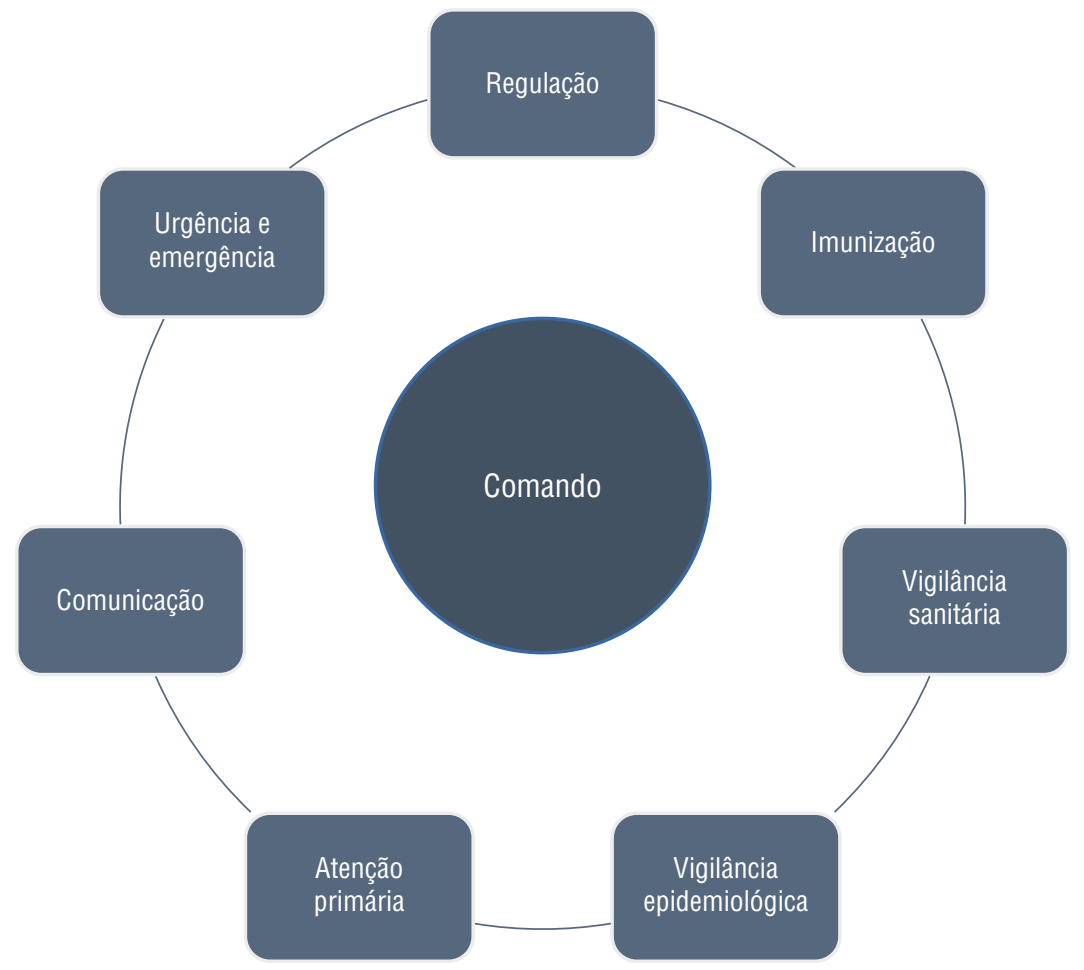

Fonte: https://coronavirus.rio/wp-content/uploads/2021/03/PlanoContingencia_v12.pdf Elaborado pelos técnicos do COE COVID-19 RIO.

a A coordenação das atividades neste centro esteve a cargo do comando, composto por um coordenador geral, grupo de operações e grupo de análises. Pontos focais pertencentes a setores chaves da SMS-Rio integraram-se ao COE COVID-19 RIO desenvolvendo atividades específicas.

Atividades de fiscalização de estabelecimentos comerciais na cidade, executadas pela Secretaria de Ordem Pública e pela vigilância sanitária, para verificação do cumprimento das medidas restritivas de proteção à vida, foram continuamente monitoradas.

Em relação às articulações interinstitucionais, o COE COVID19 RIO fortaleceu os fluxos de coleta e encaminhamento de amostras aos laboratórios de referência para sequenciamento genômico do SARS-CoV-2. Na cidade, o monitoramento das variantes foi realizado por meio das estratégias sentinela e ampliada. Pela estratégia sentinela, semanalmente foram coletadas cinco amostras de síndrome gripal (SG) e cinco amostras de síndrome respiratória aguda grave (SRAG), posteriormente encaminhadas para sequenciamento genético. Já na estratégia ampliada, 128 amostras positivas (por real time polymerase chain reaction, RT-PCR) selecionadas aleatoriamente foram direcionadas para identificação. Esses resultados seguiram periodicamente dos laboratórios à Coordenação de Informação Estratégica em Vigilância em Saúde e foram compartilhados no COE COVID-19 RIO.

Neste ponto, reitera-se a intensa colaboração da OPAS durante todo o processo de concepção e implantação do COE COVID-19 RIO, transferindo expertise técnica para a organização e coordenação da resposta.

\section{INFORMAÇÕES EM SAÚDE E APOIO DECISÓRIO}

O acesso a informações oportunas e corretas para embasar a tomada de decisões é um elemento essencial para a coordenação efetiva da resposta a emergências em saúde pública.
Nessa perspectiva, o COE COVID-19 RIO valorizou o uso dos registros dos sistemas de informação, introduzindo inteligência epidemiológica nas análises de dados.

A informação produzida no centro foi consolidada em boletins epidemiológicos que continham indicadores de incidência, mortalidade e atendimentos nas portas de entrada de serviços de saúde. Mapas e avaliação de risco nas regiões administrativas da cidade, monitoramento de novas variantes e informações sobre a campanha de vacinação também foram incluídos nesse material. Para a produção desses boletins, contínuos esforços relacionados à automatização do processamento de dados foram realizados, sendo crescente o interesse na discussão de metodologias orientadas à correção de atrasos de notificação dos casos.

Outra iniciativa para aumentar a transparência em relação aos dados da doença foi a reformulação do painel municipal da COVID-19 nas primeiras semanas de operação do COE COVID-19 RIO. Essa medida fomentou o acompanhamento da situação epidemiológica pela população; o painel pode ser acessado por meio eletrônico (https:/ / experience.arcgis.com/ experience/38efc69787a346959c931568bd9e2cc4).

Da análise crítica da informação gerada a partir da utilização dos dados dos sistemas de informação oficiais, percebeu-se a importância dos registros complementares advindos da assistência para a organização das estratégias de enfrentamento. Sob gerência dos setores de urgência e emergência, foram consolidados os registros administrativos sobre atendimentos de SG e SRAG em unidades hospitalares, unidades de pronto atendimento e centros de emergência regionais da cidade. As solicitações de vaga zero para pacientes atendidos nas unidades da rede de assistência da cidade também foram uma fonte de 
informação, advinda do complexo regulador. Esses conjuntos de dados não configuram sistemas oficiais, mas sua utilização trouxe maior sensibilidade para a identificação de tendências na incidência da doença, auxiliando a previsão de cenários epidemiológicos e o planejamento das ações de enfrentamento.

Além das iniciativas citadas anteriormente, técnicos do COE COVID-19 RIO estruturaram um projeto para avaliação dos efeitos da pandemia de COVID-19 na saúde da população com base em registros dos sistemas de informação. Essa iniciativa visa a implementação do monitoramento de indicadores de saúde no município do Rio de Janeiro, identificando oportunidades de melhoria nos processos de trabalho da vigilância em saúde principalmente frente a processos epidêmicos — para orientação de ações de saúde que previnam novos casos e mortes precoces.

\section{COMUNICAÇÃO DE RISCO}

Durante as emergências em saúde pública, é fundamental delinear estratégias de comunicação de risco direcionadas para o aumento do engajamento da população na mitigação da transmissão da doença. No COE COVID-19 RIO, a divulgação ativa do conteúdo dos boletins epidemiológicos em coletivas de imprensa semanais, transmitidas pelas redes sociais e com a participação de representantes da alta gestão, foi uma intervenção essencial na condução da resposta à pandemia. Essa iniciativa reduziu a desinformação da população ao comunicar, em formato sintetizado e oportuno, a situação sanitária da cidade.

A assessoria de comunicação atuou ativamente nas redes sociais para esclarecimento de dúvidas da população em função da infodemia gerada pela incerteza associada à doença. Destaca-se aqui a divulgação de conteúdo relativo às medidas não farmacológicas para a redução da disseminação do vírus e a publicização contínua do calendário de vacinação em vigência na cidade, visando aumentar os níveis de confiança e adesão da população às orientações contra a COVID-19.

Para o atendimento de demandas de imprensa, foi sistematizado um canal com o ponto focal da assessoria de comunicação do COE COVID-19 RIO. Esse ponto focal recebia e organizava, em conjunto com as outras áreas técnicas do COE, as respostas que foram devolvidas à imprensa. Essa validação junto aos pontos focais antecipa a comunicação de incertezas, abrandando mal-entendidos em relação às estratégias de prevenção e controle implantadas.

\section{CONSIDERAÇÕES FINAIS}

A pandemia de COVID-19 constitui-se como a maior emergência sanitária já enfrentada mundialmente, tanto pelas incertezas de uma nova doença de transmissão respiratória quanto pelas vulnerabilidades e desigualdades apresentadas pelos diferentes territórios. Esse cenário requer mudanças no processo de trabalho rotineiro, exigindo uma resposta unificada, coordenada e de comando único.

O planejamento do COE COVID-19 RIO envolveu organização de recursos humanos e físicos; atenção a aspectos chaves para a garantia de governança da resposta, como a identificação de especialistas para apoio consultivo; delineamento de um plano de ação com o detalhamento da operação pretendida; e a organização de um plano de contingência, em consonância com o modelo preconizado pela OMS.

Da experiência de implantação desse modelo, ressalta-se a sinergia alcançada a partir das articulações entre diferentes áreas técnicas - fomentadas pela existência do $\mathrm{COE}$ - e que se refletiram em avanços na vigilância da doença, a exemplo das investigações de surtos e a implementação da vigilância genômica na cidade. Reitera-se a identificação de potencialidades nos conjuntos de dados advindos das unidades de urgência e emergência, que ofereceu maior oportunidade na captação da demanda dos serviços de saúde da cidade e, consequentemente, na tomada de decisão pelos gestores.

A coordenação das ações, a partir da implantação do COE COVID-19 RIO, fomentou integração entre as áreas técnicas, inovação nas análises epidemiológicas apoiadas em fontes de informação complementares, parcerias institucionais e transparência sobre as medidas adotadas. Por fim, destaca-se a potência dessa metodologia de gestão como um legado para resposta a futuras emergências em saúde pública no município do Rio de Janeiro.

Contribuição dos autores. Todos os autores participaram da concepção deste manuscrito. DMOC contribuiu para o delineamento dos eixos da experiência, contextualização das iniciativas em consonância com o documento norteador da implantação, escrita do trabalho e revisões executadas. Todos os autores leram e aprovaram a versão encaminhada para publicação.

Agradecimentos. Aos profissionais de saúde do Município do Rio de Janeiro, incluindo os técnicos de atividades de apoio das unidades assistenciais, e àqueles que colaboraram para a estruturação do Centro de Operações de Emergência COVID-19 RIO.

Conflitos de interesse. Nada declarado pelos autores.

Declaração. As opiniões expressas no manuscrito são de responsabilidade exclusiva dos autores e não refletem necessariamente a opinião ou política da RPSP/PAJPH ou da Organização Pan-Americana da Saúde (OPAS).

\section{REFERÊNCIAS}

1. Brasil, Ministério da Saúde, Secretaria de Vigilância em Saúde. Infecção humana pelo novo coronavírus (2019-nCoV). Bol Epidemiológico. 2020:1-23. Disponível em: https://portalarquivos2. saude.gov.br/images/pdf/2020/fevereiro/07/BE-COE-Coronavirus-n020702.pdf Acessado em 13 de julho de 2021.

2. Ranzani OT, Bastos LSL, Gelli JGM, Marchesi JF, Baião F, Hamacher S, et al. Characterisation of the first 250,000 hospital admissions for COVIDCOVID-19 in Brazil: a retrospective analysis of nationwide data. Lancet Respir Med. 2021;9(4):407-18.
3. Castro MC, Kim S, Barberia L, Ribeiro AF, Gurzenda S, Ribeiro KB, et al. Spatiotemporal pattern of COVID-19 spread in Brazil. Science. 2021;372(6544):821-6.

4. Faria NR, Mellan TA, Whittaker C, Claro IM, Candido DS, Mishra S, et al. Genomics and epidemiology of the P.1 SARS-CoV-2 lineage in Manaus, Brazil. Science. 2021;372(6544):815-21.

5. Fujino T, Nomoto H, Kutsuna S, Ujiie M, Suzuki T, Sato R, et al. Novel SARS-CoV-2 variant in travelers from Brazil to Japan. Emerg Infect Dis. 2021;27(4):1243-5. 
6. Brasil, Ministério da Saúde, Secretaria de Vigilância em Saúde, Departamento de Vigilância em Saúde Ambiental e Saúde do Trabalhador. Plano de resposta às emergências em saúde pública. Brasília: Ministério da Saúde, 2014. Disponível em: https://bvsms.saude. gov.br/bvs/publicacoes/plano_resposta_emergencias_saude_ publica.pdf Acessado em 13 de julho de 2021.

7. World Health Organization (WHO). A systematic review of public health Emergency Operations Centres (EOC). Genebra: WHO; 2013. Disponível em: http:/ /apps.who.int/iris/bitstream/handle/10665/ 99043 /WHO_HSE_;jessionid=2605B2EF7BE919F816BE947E19F2F25A? sequence $=1$ Acessado em 13 de julho de 2021.

8. World Health Organization (WHO). International health regulations. $2^{a}$ ed. Genebra: WHO; 2005. Disponível em: https://www. gov.br/anvisa/pt-br/assuntos/paf/regulamento-sanitario-internacional/arquivos/7179json-file-1 Acessado em: 08/10/2021.
9. Croda J, de Oliveira WK, Frutuoso RL, Mandetta LH, Baia-da-Silva DJ, Brito-Sousa JD, et al. COVID-19 in Brazil: advantages of a socialized Unified Health System and preparation to contain cases. Rev Soc Bras Med Trop. 2020;53: e20200167.

10. World Health Organization (WHO). Public Health Emergency Operations Centre Network. Framework for a public health emergency operations centre. Genebra: WHO; Nov 2015; Disponível em: https://apps.who.int/iris/rest/bitstreams/874073/retrieve Acessado em: 14/10/2021.

Manuscrito submetido em 19 de julho de 2021. Aceito em versão revisada em 19 de outubro de 2021.

\section{COVID-19 emergency operations center: experience of the Rio de Janeiro municipality, Brazil}

\section{ABSTRACT}

Keywords

\begin{abstract}
The present report describes the implementation of an emergency operations center to coordinate the response to the COVID-19 pandemic in the municipality of Rio de Janeiro, Brazil. Following the public health emergency management framework proposed by the World Health Organization (WHO), this temporary center (COE COVID-19 RIO) started operating in January 2021. The report is organized along five themes: legal framework; structure, planning, and procedures; institutional articulation; health information for decision-making; and risk communication. Major advances obtained with the initiative include improvements in governance for the management of COVID-19, increase in the synergy among sectors and institutions, improved information sharing in relation to COVID-19 prevention and control measures, innovation in epidemiologic analyses, and gains in transparency and decision-making opportunities. In conclusion, even if conceived at an advanced stage of the pandemic in the municipality of Rio de Janeiro, the COE COVID-19 RIO has played a relevant role in shaping the city's responses to the pandemic. Also, despite its temporary character, the experience will leave a lasting legacy for the management of future public health emergencies in the municipality of Rio de Janeiro.
\end{abstract}

Emergency operations center; COVID-19; surge capacity.

\section{Centro de operaciones de emergencia en respuesta a la COVID-19: la experiencia del municipio de Rio de Janeiro}

RESUMEN En el presente artículo se describe la experiencia al establecerse un centro de operaciones de emergencia (COE) para coordinar la respuesta a la pandemia de COVID-19 en el municipio de Rio de Janeiro (Brasil). Siguiendo el modelo de gestión de emergencias de salud pública promovido por la Organización Mundial de la Salud (OMS), este centro temporal se activó en enero del 2021. El informe se estructuró con base en cinco ejes temáticos: marco legal; estructura, planes y procedimientos; articulaciones institucionales; información en materia de salud para sustentar las decisiones; y comunicación sobre riesgos. Entre los principales avances relacionados con esta iniciativa cabe destacar los adelantos en cuanto a la gobernanza para organizar la forma de enfrentar la COVID-19, el aumento de la sinergia entre los sectores y las instituciones correspondientes, un mayor intercambio de información sobre las medidas de prevención y control de la enfermedad, innovación en los análisis epidemiológicos, mayor transparencia en la toma de decisiones y decisiones tomadas de manera más oportuna. Se llegó a la conclusión de que este COE, a pesar de que había sido establecido en una fase avanzada de la pandemia en la ciudad, tuvo un papel importante en la estructuración de la respuesta. Sin embargo, a pesar de su carácter temporal, la experiencia demostró ser un importante legado para enfrentar futuras emergencias de salud pública en el municipio de Rio de Janeiro.

Palabras clave Centro de operaciones de emergencia; COVID-19; capacidad de reacción. 